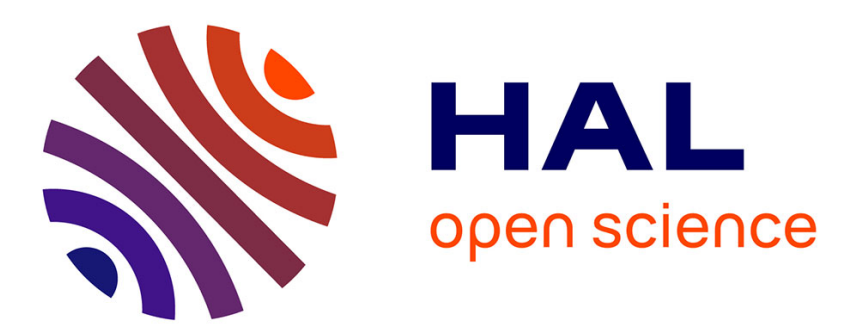

\title{
Model order reduction approach to one-dimensional collisionless closure problem
}

Camille Gillot, Guilhem Dif-Pradalier, Xavier Garbet, Philippe Ghendrih, Virginie Grandgirard, Yanick Sarazin

\section{- To cite this version:}

Camille Gillot, Guilhem Dif-Pradalier, Xavier Garbet, Philippe Ghendrih, Virginie Grandgirard, et al.. Model order reduction approach to one-dimensional collisionless closure problem. 2021. hal-03099863

\section{HAL Id: hal-03099863 https://hal.science/hal-03099863}

Preprint submitted on 6 Jan 2021

HAL is a multi-disciplinary open access archive for the deposit and dissemination of scientific research documents, whether they are published or not. The documents may come from teaching and research institutions in France or abroad, or from public or private research centers.
L'archive ouverte pluridisciplinaire HAL, est destinée au dépôt et à la diffusion de documents scientifiques de niveau recherche, publiés ou non, émanant des établissements d'enseignement et de recherche français ou étrangers, des laboratoires publics ou privés. 


\title{
Model order reduction approach to one-dimensional collisionless closure problem
}

\author{
C. Gillot, ${ }^{1,2, \text { a) }}$ G. Dif-Pradalier, ${ }^{1, \text { b) }}$ X. Garbet, ${ }^{1}$ P. Ghendrih, ${ }^{1}$ V. Grandgirard, ${ }^{1}$ and Y. Sarazin ${ }^{1}$ \\ 1) CEA, IRFM, F-13108 Saint-Paul-lez-Durance, France \\ ${ }^{2)}$ École des Ponts ParisTech, F-77455 Champs sur Marne, France
}

(Dated: 18th October 2020)

The problem of the fluid closure for the collisionless linear Vlasov system is investigated using a perspective from control theory and model order reduction. The balanced truncation method is applied to the 1D-1V Vlasov system. The first few reduction singular values are well-separated, indicating potentially low-dimensional dynamics. To avoid largedimensional numerical work, a reduced model is formulated using rational interpolation, generalising the seminal work from Hammett and Perkins. The resulting models are found to outperform the state-of-the-art models for thermal phase velocities. Thanks to the versatility of this formulation, an application to toroidal gyrokinetic dynamics is discussed.

\section{INTRODUCTION}

First-principle simulation of plasma turbulence faces a double challenge of cost and complexity. The physical and intellectual complexity stem from the non-linearity of the turbulent dynamics. The cost is induced by the kinetic nature of the instability drive. Simulating the gyrokinetic description of tokamak turbulence is now routine for numerous codes, but requires maintaining high-performance codes running smoothly on supercomputers. While simulations get longer and longer, the amount of actually analysed information remains limited in proportion. A few fluid moments of the distribution function (density, velocity, pressure...) are extracted out of the tens of grid points used to simulate dynamics in velocity space, and the rest is discarded. Reaching more day-to-day investigation of turbulent transport requires lighter models for tractable simulations. However, the obvious fluid systems fail to simulate dynamics close to the marginal instability threshold ${ }^{1}$. Collisionless closures are a way forward to avoid this shortfall while retaining dynamics in a low-dimensional space ${ }^{2,3}$. Those so-called Landau-fluid models have been applied both to magnetohydrodynamics ${ }^{4-7}$ and to gyro-fluids 8,9 .

The collisionless closure problem arises due to the nondetermination of the fluid moment $\ell+1$ in the $\ell$-fluid reduction. The goal is to find the best linear combination to recover relevant kinetic effects, to recover the kinetic response $H_{\text {kin }}(\zeta)$ as a function of the phase velocity $\zeta$. Several methods have been suggested in the literature ${ }^{10}$. Asymptotic methods use a Taylor $^{2,11}$ or Padé ${ }^{12,13}$ expansion of the kinetic response function $H_{\text {kin }}$ for $\zeta \ll 1$ and $\zeta \gg 1$ to constrain the free parameters. As such, they explicitly choose an asymptotic frequency range, and are limited to it. Other authors have introduced the excitation frequency as a parameter ${ }^{14,15}$. This renders the simulation of the closed model very difficult, because strongly non-linear. These methods have an even larger number of free parameters, and rely heavily on physical choices and orderings ${ }^{11}$.

In this paper, we will discuss two different methods, namely balanced truncation ${ }^{16-18}$ and rational interpolation ${ }^{19-21}$. The balanced truncation method considers the Vlasov equation as a control system. The forcing is the electric potential, and

\footnotetext{
a)Electronic mail: camille.gillot@polytechnique.org.

b)Electronic mail: guilhem.dif-pradalier@cea.fr.
}

observables are the fluid moments. Balanced truncation develops the distribution on components ranked by their dynamical reachability and observability through moments. By construction, the balanced reduced model only depends on the ground Vlasov equation and on the chosen observables. Rational interpolation rather constructs a phenomenological model, matching the kinetic dispersion relation at well-chosen phase velocities. As such, it provides an effective generalisation of the Padé-based methods to an arbitrary set of expansion points.

In a first part, the two methods are reviewed and their connection to the usual collisionless closures are discussed. In a second part, the two methods are applied numerically to the 1D-1V Vlasov-Poisson problem. The precision of the reconstruction and temporal evolution in the Vlasov problem are compared. The influence of the model on the behaviour of the Vlasov-Poisson problem is discussed, using the Landau damping and bump-on-tail instability as benchmarks. Finally, the extensions to the toroidal gyrokinetic problem and to nonlinear simulations are discussed.

\section{REDUCTION OF THE KINETIC EQUATION}

We consider the linear one-dimensional electrostatic Vlasov problem

$$
\partial_{t} f+i k v f=i k \mathcal{F}^{\prime} \phi
$$

where $k$ is the spatial wave-number, $\mathcal{F}^{\prime}(v)$ is the derivative of the equilibrium distribution function with respect to the velocity, $f(v)$ the fluctuation of the distribution function, $\phi$ the electrostatic potential. The velocity is normalised to the thermal velocity, and the potential to the thermal potential. Without loss of generality, we will only consider one value of $k>0$, the other cases can be deduced by symmetry.

We consider a choice of observable quantities, for instance a few fluid moments like the density $n$, the velocity $u$, the pressure $p$. The electric potential $\phi$ is considered as an input, unconstrained by Poisson equation, and forces the dynamics of the state $f$. The mapping from the electric potential $\phi$ to these moments is a linear time-invariant dynamical system. This mapping corresponds to an open-loop control system. The eventual Poisson equation provides a closed-loop condition, feeding back the observable $n$ into the input $\phi$. Control theory 
and model order reduction theory have developed useful tools for analysing the structure of such systems, and for constructing reduced-order models matching such an input-output relation $\phi \mapsto(n, u, p)$, without solving for the full state space, here the distribution function $f^{22}$.

The frequency response of the density from the Vlasov dynamics can be computed analytically as

$$
n(\zeta)=\phi(\zeta) \int_{-\infty}^{+\infty} \frac{\mathcal{F}^{\prime}(v)}{v-\zeta-i \frac{\sigma}{|k|}} \mathrm{d} v
$$

with $\zeta=\omega /|k|$ the phase velocity of the perturbation, normalised to the thermal velocity. We have introduced a small damping rate $\sigma$. The integral on the right-hand-side is singular if $\sigma=0$. The so-called Landau prescription enables to evaluate it for positive damping rate $\sigma$. This corresponds to the Laplace transform formalism, and enforces causality. The resulting $n / \phi$ function is holomorphic, and can be extended to the negative complex plane.

When the equilibrium distribution function is a Maxwellian, the frequency response if expressed using the Fried and Conte function $Z^{23-25}$.

$$
H_{\mathrm{kin}}(\zeta)=\frac{n}{\phi}=-1-\frac{\zeta}{\sqrt{2}} \mathcal{Z}\left(\frac{\zeta}{\sqrt{2}}\right)
$$

\section{THE FLUID HIERARCHY TRUNCATION}

In order to reduce the simulation cost, we need a reduction step. The traditional method involves the formulation of the fluid hierarchy. It consists in the projection of the Vlasov equation (1) against graded polynomials. For instance, the fluid moments $m_{\ell}$ verify

$$
\begin{aligned}
m_{\ell} & =\int v^{\ell} f \mathrm{~d} v \\
\partial_{t} m_{\ell}+i k m_{\ell+1} & =-i k \phi \ell \int v^{\ell-1} \mathcal{F} \mathrm{d} v
\end{aligned}
$$

where $m_{0}$ is the density $n, m_{1}$ is the momentum $N_{\text {eq }} u, m_{2}$ is the pressure $p$. The equation for each moment $m_{\ell}$ requires the one for the next moment, ad infinitum. Practical applications require to truncate this hierarchy somehow.

The last relevant moment has to be expressed using the available information. In the linear setting, it is just a linear combination of its forebearers and of the forcing $\phi$. Once the fluid hierarchy is closed, the frequency response can be constructed, and compared to the kinetic response $H_{\text {kin }}$. One of the simplest closure ansatz corresponds to putting the integral of the $\ell+1^{\text {st }}$ Hermite polynomial to zero. Other definitions are possible, like setting the $\ell+1^{\text {st }}$ cumulant to zero ${ }^{10}$. In the linear setting, both are equivalent. For instance, this 4-field fluid model writes

$$
\begin{aligned}
\int\left(v^{4}-6 v^{2}+3\right) f \mathrm{~d} v & \approx 0 \\
H_{\text {Fluid } 4}(\zeta) & =\frac{\zeta^{2}-3}{\zeta^{4}-6 \zeta^{2}+3}
\end{aligned}
$$

This transfer function is real, all its poles are on the real axis, so the model is conservative: it is not able to reproduce a damping mechanism as in the kinetic case. It should be noted that even- and odd-dimensional models in this family behave differently. Even-dimensional models correctly reproduce the adiabatic response $n=-\phi$ for $\omega \rightarrow 0$. Odd-dimensional models are more precise at high frequency, at the expense of an incorrect adiabatic response. For instance, the 5-dimensional fluid model writes

$$
\begin{aligned}
\int\left(v^{5}-10 v^{3}+15 v\right) f \mathrm{~d} v & \approx 0 \\
H_{\text {Fluid } 5}(\zeta) & =\frac{\zeta^{2}-7}{\zeta^{4}-10 \zeta^{2}+15}
\end{aligned}
$$

It correctly decays asymptotically as $1 / \zeta^{2}$, but the adiabatic response is $H(\zeta=0)=-7 / 15$ instead of the correct -1 .

\section{REVIEW OF THE BALANCED TRUNCATION METHOD}

Balanced truncation allows approaching the model reduction problem as a linear optimisation problem ${ }^{18}$. Instead of a term-by-term matching of some expansion, it can be thought as a uniformly-weighted matching. This method is systematic, and only depends on (i) the original model equation, (ii) an energy functional on the input variables and (iii) a quadratic functional on the output variables. The reduced model is constructed by removing hard-to-reach and hard-to-observe states from the dynamics.

\section{A. Notions of reachability and observability}

Given a potential perturbation $\phi(t)$, the response of the distribution function is given by

$$
\begin{aligned}
f(t, v) & =\int g_{\tau}(v) i k \phi(t-\tau) \mathrm{d} \tau \\
g_{\tau}(v) & =\mathrm{e}^{-i k v \tau-\sigma \tau} \mathcal{F}^{\prime}(v)
\end{aligned}
$$

$g$ defines an infinite family of distribution functions, indexed by $\tau$. All solutions to equation (1) are superpositions inside this family of states, as given by equation (10). From the opposite point of view, let $f(v)$ be an arbitrary distribution function. The easier $f$ is to represent as a linear combination of $g_{\tau}$, the closer it is to an actual solution of (1). Conversely, the more energy $k^{2}|\phi|^{2}$ a state requires to appear, the more convoluted $\phi$ is in (10), the less reachable it is.

To quantify this, we reduce the infinite family $g$ to a set of principal components ${ }^{26}$. In order to achieve this, we construct the so-called reachability Gramian $\mathcal{R}$

$$
\begin{aligned}
\mathcal{R}\left(v^{\prime}, v^{\prime \prime}\right) & =\int_{0}^{+\infty} g_{\tau}\left(v^{\prime}\right) g_{\tau}^{*}\left(v^{\prime \prime}\right) \mathrm{d} \tau \\
& =\frac{\mathcal{F}^{\prime}\left(v^{\prime}\right) \mathcal{F}^{\prime}\left(v^{\prime \prime}\right)}{2 \sigma+i k\left(v^{\prime}-v^{\prime \prime}\right)}
\end{aligned}
$$


This (infinite-dimensional) matrix is symmetric, positive and bounded. It can be diagonalised as

$$
\mathcal{R}\left(v^{\prime}, v^{\prime \prime}\right)=\sum_{p=0}^{+\infty} \lambda_{p}^{2} f_{p}\left(v^{\prime}\right) f_{p}^{*}\left(v^{\prime \prime}\right)
$$

The orthonormal eigenvectors $f_{p}$ correspond to principal components. The eigenvalues $\lambda_{p}^{2}$ allow to order them from most reachable (high $\lambda_{p}$ ) to least reachable (low $\lambda_{p}$ ). All the distributions functions in the family $g_{\tau}$ can be written as an (infinite) sum of $f_{p}$. For numerical application, we will discretise the velocity space. The Gramian $\mathcal{R}$ will become a matrix $\mathcal{R}_{p q}=\mathcal{R}(p \Delta v, q \Delta v)$.

To diagonalise, we need the $f_{p}$ to be orthogonal, but we are yet define with which inner product. We need a second symmetric matrix to define this orthogonality. It can be provided by measuring the observability of the states. Given a distribution function $f$ at $t=0$, the density at later times is given by

$$
n(t)=\int \mathrm{e}^{-i k v t-\sigma t} f(v) \mathrm{d} v
$$

A state that cannot be observed is of no interest for us. Conversely, a state that is clearly and strongly visible for a long time is physically relevant and should be captured. For our toy model, we chose to only measure the density response $n$. The signal strength for a state $f$ can be measured as a squared norm of $n$ for positive times. We introduce the observability Gramian $O$

$$
\begin{aligned}
\int_{0}^{+\infty}|n(t)|^{2} \mathrm{~d} t & =\int O\left(v^{\prime}, v^{\prime \prime}\right) f^{*}\left(v^{\prime}\right) f\left(v^{\prime \prime}\right) \mathrm{d} v^{\prime} \mathrm{d} v^{\prime \prime} \\
O\left(v^{\prime}, v^{\prime \prime}\right) & =\frac{1}{2 \sigma-i k\left(v^{\prime}-v^{\prime \prime}\right)}
\end{aligned}
$$

As a symmetric positive matrix, $O$ provides an inner product. We can proceed to computing our principal components equation (13). In this case, $\lambda_{p}$ is called a Hankel singular value. The orthonormality of the $f_{p}$ makes $\lambda_{p}^{2}$ scale with their observability. Therefore, the $\lambda_{p}$ measure the product of observability and reachability for a distribution component $f_{p}$. Since the components are orthogonal with respect to $O$, we can construct the dual basis $\mu_{p}$

$$
\begin{aligned}
\mu_{p}\left(v^{\prime}\right) & =\int O\left(v^{\prime}, v^{\prime \prime}\right) f_{p}\left(v^{\prime \prime}\right) \mathrm{d} v^{\prime \prime} \\
\int \mu_{p}(v) f_{q}(v) \mathrm{d} v & =\delta_{p q}
\end{aligned}
$$

\section{B. Usage for model order reduction}

We decompose the perturbed distribution function on the basis defined by the $f_{p}$, weighted by new dynamical variables $x_{p}$. The functions $\mu_{p}$ give a projection basis, a set of general- ised moments to consider for the reduction.

$$
\begin{aligned}
f(t, v) & =\sum_{p=0}^{+\infty} x_{p}(t) f_{p}(v) \\
x_{p}(t) & =\int \mu_{p}(v) f(t, v) \mathrm{d} v
\end{aligned}
$$

By plugging this form into the Vlasov equation (1) and projecting against the $\mu_{p}$, we can formulate the evolution of the $x_{p}$ as

$$
\begin{aligned}
\dot{x}_{p} & =\sum_{q=0}^{+\infty} A_{p q} x_{q}+B_{p} \phi \\
m_{\ell} & =\sum_{q=0}^{+\infty} C_{\ell q} x_{q}
\end{aligned}
$$

with the matrices

$$
\begin{aligned}
A_{p q} & =\iint \mathrm{d} x \mathrm{~d} v \mu_{p}(x, v)(-i k v) f_{q}(x, v) \\
B_{p} & =\iint \mathrm{d} x \mathrm{~d} v \mu_{p}(x, v) \mathcal{F}^{\prime}(v) i k \\
C_{\ell q} & =\int \mathrm{d} v v^{\ell} f_{q}(x, v)
\end{aligned}
$$

Truncating the sum in equation (19) provides a finitedimensional model, with state defined by the $x_{p}$. The openloop reduced model is guaranteed to be stable as soon as the original model is, which is the case for equation (1). However, and this will be developped below, this does not guarantee the stability of the closed-loop model, like Vlasov-Poisson.

The relevance of the truncation is defined by the sequence of Hankel singular values $\lambda_{p}$. The accuracy of the reduced model is defined by the sum of neglected singular values ${ }^{18}$. More generally, the sequence of singular values allows to estimate the reducibility of the original equation. The faster they decay, the shorter the sum (19) can be to reach the same accuracy.

This method departs from the more traditional principal component analysis ${ }^{26,27}$ by using the observability Gramian instead of the implicit identity matrix. The latter is determined by the data acquisition choice rather than physics, and leads to poorer reduced models ${ }^{28}$. We remark the scaling of the Gramians does not modify the reduced basis, making the scalar $\sigma /|k|$ the only regularisation parameter.

\section{Numerical investigation}

The kinetic equation (1) describes dynamics in a continuousvelocity space. Grid-based simulations require a discretisation in the velocity space. Because of the finite grid, the discrete system is plagued by a return to initial conditions. With a uniform radial grid of step $\Delta v$, the impulse response is written

$$
f(t, j \Delta v)=\mathrm{e}^{-i j k \Delta v t-\sigma t} i k \mathcal{F}^{\prime}(j \Delta v) \phi(t=0)
$$

The first exponential factor is periodic in time, of period $T=$ $2 \pi / k \Delta v$. This periodicity is a spurious numerical echo. It is 


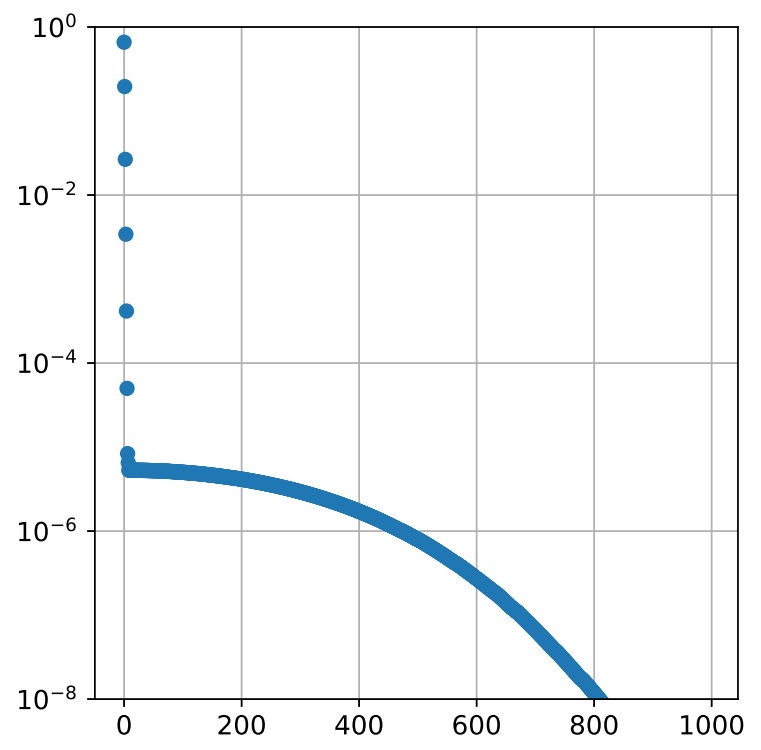

Figure 1. Hankel singular values of the balanced truncation problem.

due to the discretisation, and can be thought as aliasing in $k_{v}$ space. Relevant simulations thus require a regularisation to remove this effect, such as $\sigma \gg k \Delta v$. This can be achieved either through a thinner grid, or by strong enough collision term. However, one must care not too increase $\sigma$ too much, to avoid modifying too much the very dynamics we are trying to approximate.

We compute numerically the two Gramian matrices (12) and (16) on a uniform velocity grid between -5 and 5 times the thermal velocity, with $\Delta v=10^{-2}$. Regularisation is $\sigma / k \Delta v=$ 2. It should be noted that up to a scaling factor, the two matrices (12) and (16) only depend on $\sigma$ and $k$ through the ratio $\sigma /|k|$. As a consequence, in the following and until paragraph VIB, we shall consider only $k=1$.

We use the Schur-based computation method from Penzl ${ }^{29}$. Given the Cholesky factorisation of the matrices $\mathcal{R}=R R^{\dagger}$ and $O=O O^{\dagger}$, the singular value decomposition of $R^{\dagger} O$ is computed. The singular values are exactly the Hankel singular values $\lambda_{p}$. The left singular vectors $u_{p}$ yield the distribution components $f_{p}=R u_{p}$.

Hankel singular values are plotted figure 1. The figure outlines seven outstanding well-separated singular values and a bulk of singular values with Gaussian decrease. The first seven correspond to truncation relevant modes. The bulk modes are a reminder of Van-Kampen modes around each velocity grid point. These actually are purely numerical components, due to the finite-time recurrence of the Fourier-space formulation. Increasing $\sigma / \Delta v$ decreases the bulk, and unearthes additional discrete modes.

The strong separation between the first singular values is promising for model order reduction perspectives. In our case, the singular values drop 5 orders of magnitude in the first 7 singular values. The relevance of additional dimensions for the description decays rapidly. From this consideration,
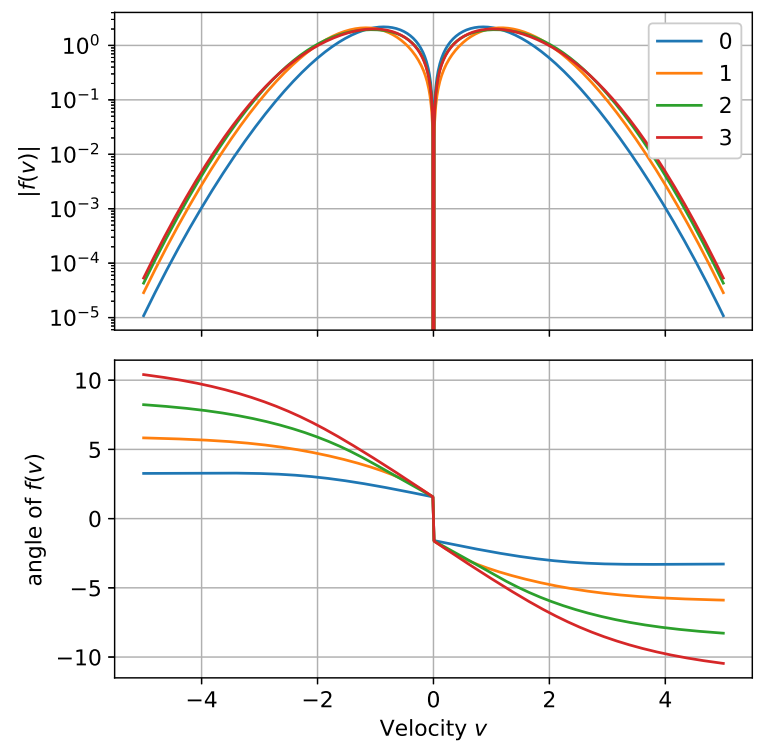

Figure 2. Plot of the components corresponding to the first four Hankel singular values. Above: norm of the distribution components $f_{k}(v)$. Below: complex angle of the distribution components $f_{k}(v)$.

the dynamics of the linear Vlasov equation (1) appears to be low-dimensional, and a reduced model should be accessible. Conversely, the singular values corresponding to the bulk are very close together. Adding components $f_{p}$ from this bulk should not increase the accuracy of a reduced model.

The balanced truncation basis is shown figure 2 . The structures are different from the Hermite polynomial used for fluid modelling. The complex amplitude of the components $f_{p}$ is reminiscent of the derived equilibrium $\left|f_{p}\right| \sim \mathcal{F}^{\prime}(v)$. Surprisingly, the complex argument of the $f_{p}(v)$ decreases monotonically with $v$. This indicates that only negative velocity-Fourier wave-numbers $k_{v}<0$ are involved.

Indeed, the filamentation process appears as an advection in velocity-Fourier space: $k_{v}=k_{v}(t=0)+k t$. Meanwhile, polynomial moments observe the neighbourhood of $k_{v}=0$ : $m_{\ell}=\left(-i \partial_{k_{v}}\right)^{\ell} f(0)$. For a mode with $k_{v}>0$ at $t=0$, the value of $k_{v}$ drifts towards $k_{v} \rightarrow+\infty$, and the mode is never observed. Conversely, negative $k_{v}$ modes pass once by $k_{v}=0$, are observed, before going away at $k_{v} \rightarrow+\infty$.

\section{REVIEW OF INTERPOLATION-BASED REDUCTION}

Balanced truncation requires to construct two Gramian matrices. For a velocity grid of size $N_{v}$, each Gramian has size $N_{v}^{2}$. This renders the method computationally expensive. Furthermore, the performances of the method are bounded by those of the discrete Vlasov model.

On the contrary, interpolation-based model order reduction is a phenomenological method. It only requires the evaluation of the reference kinetic frequency response function $H_{\text {kin }}$, equation (3). The closure problem is a reduction of the infinite-dimensional Vlasov equation to a finite dimensional 
dynamical system whose variables are the fluid moments. Because of the finite dimension, the associated transfer function $n / \phi$ is a rational function of the phase velocity $\zeta$, akin to equation (7). Instead of constructing a projection basis equations (19-20) for the Vlasov equation, we can try to directly approximate the reduced transfer function. Several methods have been employed to this end ${ }^{22,30}$. We chose to present the interpolation framework ${ }^{31,32}$ : how to construct a rational transfer function $H_{\text {red }}$ which interpolates $H_{\text {kin }}$ at chosen points?

This can be done using the so-called Loewner framework ${ }^{33}$. The method has found wide applications, not limited to model order reduction $^{34-38}$. First, let us generalise the problem. Our reduced linear time-invariant system (equation (21)) can be written in the so-called descriptor form as

$$
\begin{aligned}
E \dot{x} & =A x+B \phi \\
n & =C x
\end{aligned}
$$

where $E$ and $A$ are two square matrices, where $E$ is invertible. $B$ is a column matrix, and $C$ is a row matrix. $x$ is the state variable. The associated response function is

$$
H_{\mathrm{red}}(\zeta)=C \cdot(-i \zeta E-A)^{-1} \cdot B
$$

This representation is highly redundant: changing $x$ into $S^{-1} x$ for some matrix $S$ does not change the dynamics, only the matrices $E, A, C$. Neither does left-multiplying the equation (27) by a matrix $T$, only the matrices $E, A, B$. This freedom allows to chose the coefficients in $B$ and $C$, and then to fill-up the matrices $E$ and $A$.

\section{A. Construction of the matrices}

We want to construct a set of matrices $E, A, B, C$ such that $H_{\text {red }}\left(\alpha_{p}\right)=H_{\text {kin }}\left(\alpha_{p}\right)$ and $H_{\text {red }}\left(\beta_{q}\right)=H_{\text {kin }}\left(\beta_{q}\right)$ for two sets of (possibly complex) frequencies $\alpha_{p}$ and $\beta_{q}$. We chose to define arbitrarily $B_{p}$ and $C_{q}$ to the interpolated values. The response function $H_{\text {red }}$ will interpolate $H_{\text {kin }}$ as soon as the inverted matrix $-i \zeta E-A$ allows to select the right coefficient off $B_{p}$ or $C_{q}$. This can be achieved by constructing the two matrices $E$ and $A$ such that, for all $p, q$,

$$
\begin{aligned}
\left(-i \alpha_{p}\right) E_{p q}-A_{p q} & =C_{q} \\
\left(-i \beta_{q}\right) E_{p q}-A_{p q} & =B_{p} \\
B_{p} & =H_{\text {kin }}\left(\alpha_{p}\right) \\
C_{q} & =H_{\text {kin }}\left(\beta_{q}\right)
\end{aligned}
$$

The first equation is our selection rule: $C$ must be the $p^{\text {th }}$ row of $-i \alpha_{p} E-A$. This guarantees that $H_{\text {red }}\left(\alpha_{p}\right)=C$. $\left(-i \alpha_{p} E-A\right)^{-1} \cdot B=B_{p}$. Likewise, the second equation forces $H_{\text {red }}\left(\beta_{q}\right)=C_{q}$. Finally, the definitions of $B$ and $C$ give the values to be interpolated.

By modifying equations (30-33), the method can be generalised to also interpolate the derivatives ${ }^{33}$ and to match the asymptotic behaviour at high frequency. In order to interpolate the point $\alpha_{p}$ up to order $K$, the trick is to arrange the successive derivatives of $H_{\text {red }}$ in order after the value. The matrix $C$ is such that, for $0 \leqslant r \leqslant K$,

$$
\begin{aligned}
C_{q+r} & =\frac{1}{i^{r} r !} H_{\mathrm{red}}^{(r)}\left(\beta_{q}\right) \\
& =C \cdot\left[\left(-i \beta_{q} E-A\right)^{-1} \cdot E\right]^{r} \cdot\left(-i \beta_{q} E-A\right)^{-1} \cdot B
\end{aligned}
$$

By straightforward recursion, this is equivalent to asserting, in addition to (30), that for $1 \leqslant r \leqslant K$,

$$
-i \beta_{q} E_{p, q+r}-A_{p, q+r}=E_{p, q+r-1}
$$

For points at infinity, the formulation is reversed: for $1 \leqslant r \leqslant$ $K$,

$$
\begin{aligned}
H_{\text {red }}(\zeta) & =\sum_{r=0}^{+\infty} \frac{C \cdot i E^{-1} \cdot\left[i A \cdot E^{-1}\right]^{r} \cdot B}{\zeta^{r+1}} \approx \sum_{r=0}^{K} \frac{i B_{p+r}}{\zeta^{r+1}}(37) \\
E_{p, q} & =C_{q} \\
E_{p+r, q} & =i A_{p+r-1, q}
\end{aligned}
$$

Using the two last equations, we recognise the well-known form of the fluid equations (5) as

$$
\begin{aligned}
A \cdot E^{-1} & =\left(\begin{array}{cccc}
0 & -i & & \\
& \ddots & \ddots & \\
& & 0 & -i \\
\gamma_{0} & \cdots & \cdots & \gamma_{N}
\end{array}\right) \\
C \cdot E^{-1} & =\left(\begin{array}{cccc}
1 & 0 & \cdots & 0
\end{array}\right) \\
B_{p} & =-i k \int v^{p} F_{\mathrm{eq}} \mathrm{d} v
\end{aligned}
$$

where $\gamma$ gives the coefficient of the closure. We recover that any solution formulated as the closure of the $N^{\text {th }}$ moment matches the behaviour at infinity at order $N$. Two-sided interpolation " $\alpha=\beta$ " is possible, doubling the order of interpolation.

\section{B. Collisionless closures as special cases}

For instance, to compute an order $\ell$ realisation matching the high-frequency behaviour at order $2 \ell$, we consider setting $\alpha=\beta$ all infinite. If $\ell$ is even, it corresponds to setting the $\ell+1^{\text {st }}$ cumulant to zero. In this case, the Loewner matrices have anti-diagonal (Hankel) structure, and can be written as

$$
\begin{aligned}
H_{\mathrm{red}}(\zeta) & =\sum_{r \geqslant 1} \frac{i^{r} \gamma_{r}}{\zeta^{r}} \\
E & =\left(\begin{array}{ccc}
\gamma_{1} & \cdots & i^{\ell-1} \gamma_{\ell} \\
\vdots & \ddots & \vdots \\
i^{\ell-1} \gamma_{\ell} & \cdots & i^{2 \ell-2} \gamma_{2 \ell-1}
\end{array}\right) \\
A & =\left(\begin{array}{ccc}
\gamma_{2} & \cdots & i^{\ell-1} \gamma_{\ell+1} \\
\vdots & \ddots & \vdots \\
i^{\ell-1} \gamma_{\ell+1} & \cdots & i^{2 \ell-2} \gamma_{2 \ell}
\end{array}\right) \\
B=C & =\left(\begin{array}{ccc}
\gamma_{1} & \cdots & i^{\ell-1} \gamma_{\ell}
\end{array}\right)
\end{aligned}
$$

If $\ell$ is odd, the matrix $E$ is singular, the model is degenerate. In order to have the models corresponding to zeroing the $\ell^{\text {th }}$ 
cumulant, we need interpolate the adiabatic dynamics by setting $\beta_{\ell}=0$ (but keep $\alpha_{\ell}=\infty$ ). In that case, the matrices become

$$
\begin{aligned}
E & =\left(\begin{array}{cccc}
\gamma_{1} & \cdots & i^{\ell-2} \gamma_{\ell-1} & H(0) \\
i \gamma_{2} & \cdots & i^{\ell-1} \gamma_{\ell} & i \gamma_{1} \\
\vdots & \ddots & \vdots & \vdots \\
i^{\ell-1} \gamma_{\ell} & \cdots & i^{2 \ell-3} \gamma_{2 \ell-2} & i^{\ell-1} \gamma_{\ell-1}
\end{array}\right) \\
A & =\left(\begin{array}{cccc}
\gamma_{2} & \cdots & i^{\ell-2} \gamma_{\ell} & \gamma_{1} \\
\vdots & \ddots & \vdots & \vdots \\
i^{\ell-1} \gamma_{\ell+1} & \cdots & i^{2 \ell-3} \gamma_{2 \ell-1} & i^{\ell-1} \gamma_{\ell}
\end{array}\right) \\
B & =\left(\begin{array}{llll}
\gamma_{1} & \cdots & i^{\ell-1} \gamma_{\ell}
\end{array}\right) \\
C & =\left(\begin{array}{llll}
\gamma_{1} & \cdots & i^{\ell-2} \gamma_{\ell-1} & H(0)
\end{array}\right)
\end{aligned}
$$

In addition, the 3-field and 4-field Hammett-Perkins models correspond to all infinite $\alpha$, with $\beta=(\infty, 0,0)$ and $\beta=(\infty, 0,0,0)$. This choice of interpolation points directly corresponds to the Padé approximation order in ${ }^{2}$. More generally, we expect all the closures found by Hunana et al. ${ }^{12,13}$ to be computable by this method, using only high-order interpolation at 0 and $\infty$.

This interpolation method is very lightweight, and almost phenomenological: the model is built so as to match the behaviour at phase velocities $\alpha_{p}$ and $\beta_{q}$. However, the choice of those $\alpha_{p}$ and $\beta_{q}$ is completely free, and left to the wisdom of the physicist. The hydrodynamic behaviour is encoded by interpolation at infinity, while kinetic effects happen for finite phase velocities. The symmetry between positive and negative phase velocities advises using interpolation points with the same symmetry. While optimality properties have been shown for some special relations between the interpolation points ${ }^{19,20}$, this choice has not been retained here because of numerical difficulties.

\section{NUMERICAL COMPARISON WITH KNOWN CLOSURE SCHEMES}

We compute two interpolated models: a 3-dimensional model matching high-frequency behaviour at order 3 and interpolating $H_{\text {kin }}$ at $\zeta=0, \pm 1.3$; and a 4-dimensional model matching high-frequency behaviour at order 2 , interpolating at order 2 at $\zeta=0$, and at order 1 at $\zeta= \pm 1.3$ and $\zeta= \pm 7.04$. The value of 1.3 has been chosen inside the thermal region. The value of 7.04 has been chosen so as to preserve the sign of the imaginary part. Choosing symmetric interpolation points makes the model real when written in physical coordinates. Those are compared to the zero-cumulant fluid models and to Hammett-Perkins models. Wang's closure ${ }^{15}$ is equivalent to 4-field Hammett-Perkins, and is not studied separately.

$$
\begin{gathered}
H_{\text {Balanced } 3}(\zeta)=\frac{-0.014 \zeta^{2}-0.816 i \zeta+4.767}{-i \zeta^{3}+3.634 \zeta^{2}+6.886 i \zeta-4.735} \\
H_{\text {Interpolation } 3}(\zeta)=\frac{-i \zeta+2.65}{-i \zeta^{3}+2.65 \zeta^{2}+4.613 i \zeta-2.65} \\
H_{\text {Hammett-Perkins } 3}(\zeta)=\frac{-i \zeta+1.596}{-i \zeta^{3}+1.596 \zeta^{2}+3.0 i \zeta-1.596} \\
H_{\text {Fluid } 3}(\zeta)=-\frac{i \zeta}{-i \zeta^{3}+3.0 i \zeta} \\
H_{\text {Balanced } 4}(\zeta)=\frac{0.002 i \zeta^{3}+1.04 \zeta^{2}+5.529 i \zeta-17.426}{\zeta^{4}+5.928 i \zeta^{3}-17.996 \zeta^{2}-27.32 i \zeta+17.441} \\
H_{\text {Interpolation } 4}(\zeta)=\frac{\zeta^{2}+4.783 i \zeta-10.268}{\zeta^{4}+4.836 i \zeta^{3}-12.967 \zeta^{2}-17.652 i \zeta+10.268} \\
H_{\text {Hammett-Perkins } 4}(\zeta)=\frac{\zeta^{2}+3.519 i \zeta-5.615}{\zeta^{4}+3.519 i \zeta^{3}-8.615 \zeta^{2}-10.556 i \zeta+5.615} \\
H_{\text {Fluid } 4}(\zeta)=\frac{\zeta^{2}-3.0}{\zeta^{4}-6.0 \zeta^{2}+3.0}
\end{gathered}
$$

For most cases, the stationary limit $\zeta \rightarrow 0$ verifies $H \rightarrow-1$, recovering the expected adiabatic response at low-frequency $n \sim-n_{0} \times e \phi / T$. For high-frequency waves $\zeta \rightarrow \infty$, the fluid, Hammett-Perkins and interpolated formulations correctly give $H \sim \zeta^{-2}$. Balanced truncation is worse performing in this region, exhibiting a slower decrease $H \sim \alpha \zeta^{-1}+(1+\beta) \zeta^{-2}$, with $\alpha$ and $\beta$ going to 0 when increasing the number of components.

\section{A. Accuracy of the Vlasov dynamics}

The error on density are plotted figure 3 , as the complex modulus $\left|H_{\text {model }}(\zeta)-H_{\text {kin }}(\zeta)\right|$. For reference, the kinetic response $\left|H_{\text {kin }}(\zeta)\right|$ is plotted in dotted line. Adding components $f_{p}$ to the balanced model from the bulk of Van-Kampen modes does not increase accuracy. Our new models outperforms both Hammett-Perkins formulations in the thermal phase velocity region, without a significant penalty in the low phase velocity region. Unsurprisingly, the best response in the high phase velocity region is obtained from more conventional fluid models, because of very weak kinetic effects in this regime. As the interpolated models explicitly constrain their behaviour at infinity, their performances are comparable. Landau phasemixing phenomenon is embedded into the balanced truncation formulation as a damping term. This is confirmed by looking at the eigenvalues of the balanced truncation system, table I, whose imaginary part are well below the $\sigma=0.02$ regularisation term. More generally, all the eigenvalues of both the balanced truncation and interpolation model have a larger negative real part than the Hammett-Perkins model, hinting at a stronger damping behaviour. The imaginary parts come in the thermal range, and in conjugate pairs to keep the symmetry between positive and negative phase velocity. 

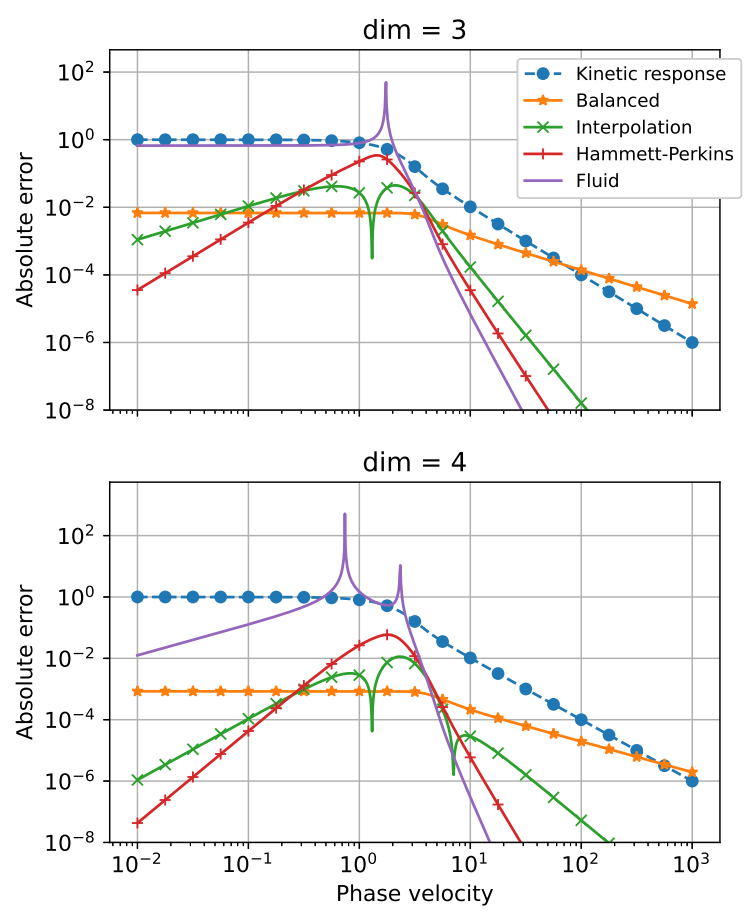

Figure 3. Reduced model error for density as a function of phase velocity. The "kinetic response" curve corresponds to $\left|H_{\text {kin }}(\zeta)\right|$, to be used as a baseline for comparison. The "balanced" curves correspond to the response of the balanced truncation model. The "fluid" curves correspond to the zero-cumulant models.

\begin{tabular}{|c|cc|}
\hline Model & \multicolumn{2}{|c|}{ Eigenvalues } \\
\hline Balanced 3 & $-1.191 i$ & $\pm 1.576-1.222 i$ \\
Interpolation 3 & $-0.863 i$ & $\pm 1.508-0.894 i$ \\
Hammett-Perkins 3 & $-0.671 i$ & $\pm 1.472-0.463 i$ \\
Fluid 3 & 0 & \pm 1.732 \\
\hline Balanced 4 & $\pm 0.678-1.485 i$ & $\pm 2.087-1.479 i$ \\
Interpolation 4 & $\pm 0.617-1.272 i$ & $\pm 1.956-1.146 i$ \\
Hammett-Perkins 4 & $\pm 0.555-1.004 i$ & $\pm 1.922-0.755 i$ \\
Fluid 4 & \pm 0.742 & \pm 2.334 \\
\hline
\end{tabular}

Table I. Eigenvalues of the reduced models.

\section{B. Coupled Vlasov-Poisson problem}

The previous section investigated the behaviour of the uncoupled Vlasov equation. We now try to recover two wellknown features of the Vlasov-Poisson system: Landau damping and bump-on-tail instability. The dispersion relation is

$$
k^{2}=H(\zeta)
$$

with $H$ one of the frequency responses above, and $k$ is the spatial wave-number scaled to the Debye length. We solve this dispersion relation by computing the right-hand-side $H(\zeta)$ on a grid of complex $\zeta$. For each value of $\mathfrak{R}[\zeta]$, seek the value of $\mathfrak{J}[\zeta]$ where the imaginary part $\mathfrak{J}[H[\zeta]]$ is closest to zero. The

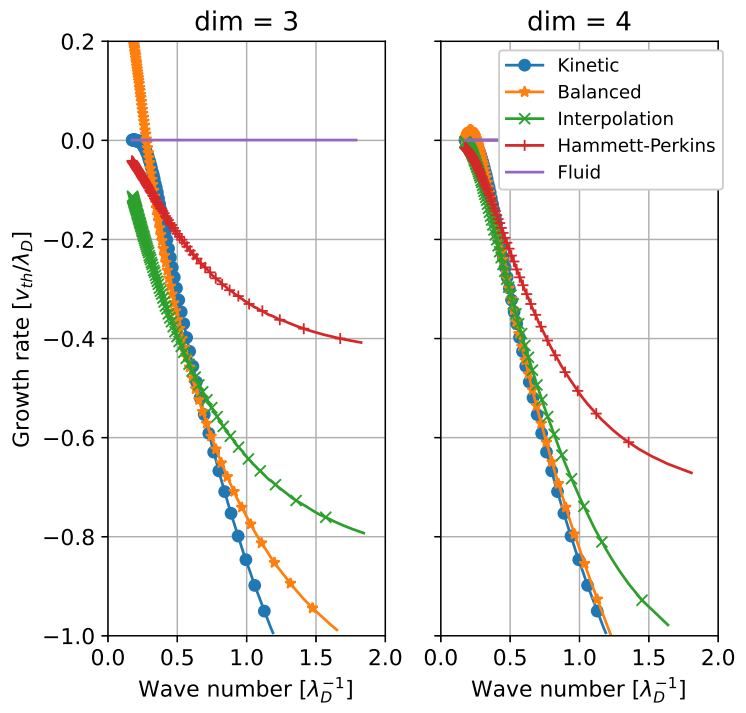

Figure 4. Numerical dispersion relations for the Landau-damping test.

value of $k^{2}$ is given by $\mathfrak{R}[H[\zeta]]$. A more elaborate method would be to solve the polynomial equations arising from the transfer functions, but precision is not an issue.

The results are given figure 4 . We observe that for $k>0.4$, the behaviour of the 4-field balanced truncation model is very close to the kinetic result. Unfortunately, a very low $k$ instability appears, related to high phase velocities. Even though the maximal growth rate is small (smaller than the regularisation $\sigma$ for the "balanced 4" model), closures produced by balanced truncation are unphysical. This is due to the aberrant behaviour of the transfer function for high phase velocities: the analytic response is closest to the positive real axis for $\zeta \rightarrow \infty$, and the reduced model tends to overshoot. The violated property is known as positivity or passivity ${ }^{22}$ : the real part of $i \zeta n / \phi$ must be positive when $\mathfrak{J}[\zeta] \geqslant 0$. This corresponds to an energy flow from the wave to the particles, so that $\vec{J} \cdot \vec{E} \geqslant 0$. Balanced-truncation itself only guarantees open-loop stability, passivity-preserving extensions of the method exist ${ }^{39}$ but pose significant numerical difficulties.

The interpolation method outperforms the HammettPerkins method in both cases. While being less precise than the balanced model for $k>0.4$, the passivity constraint is respected, and Landau damping damps. The optimal choice in terms of interpolation error should be $\alpha=\beta$ opposite complex conjugates of the dynamical poles ${ }^{20}$. Unfortunately, this choice leads to the same kind of passivity breakage.

We also benchmark the bump-on-tail instability. It can be excited by mixing another cold Maxwellian into the equilibrium. To study it, we double the state space, once for the bulk, once for the beam. We use the same method for solving the dispersion relation

$$
k^{2}=H(\zeta)+n_{1} H\left(\frac{\zeta-u_{1}}{\sqrt{T_{1}}}\right)
$$




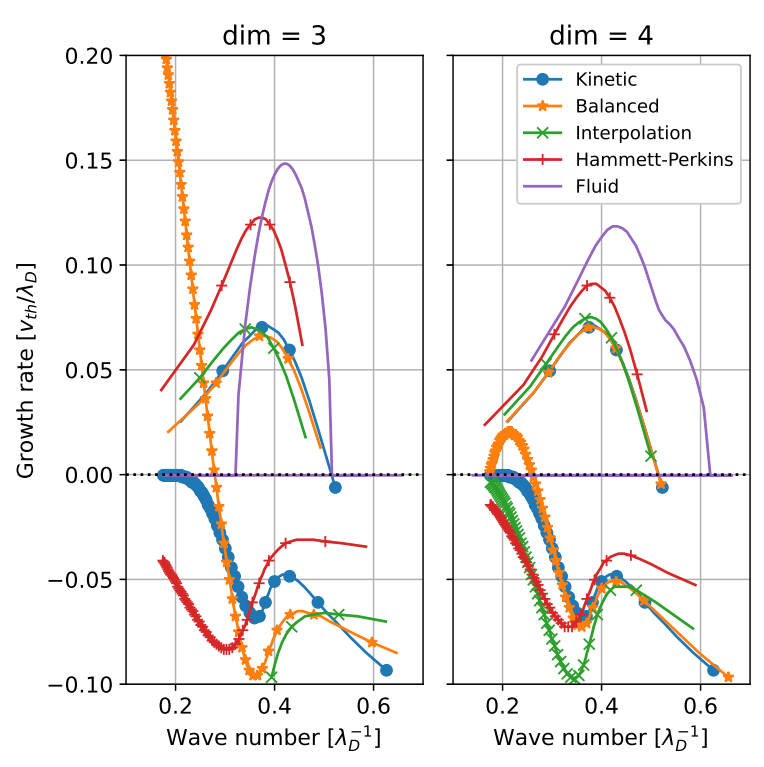

Figure 5. Numerical dispersion relations for the bump-on-tail distribution function. The bump-on-tail case uses $n_{1}, u_{1}, T_{1}=(.2,3, .01)$. The slope of the distribution function is reversed for $2.7<v<3$.

where $n_{1}=0.2, u_{1}=3$ and $T_{1}=0.01$ are the density, velocity and temperature of the cold beam. In the region where the slope of the distribution function is reversed, for $2.7<\zeta<3$, the system is unstable. Farther from this phase velocity (including opposive phase velocity), the waves are Landau-damped. The results are shown on figure 5. As a function of the wavenumber $k$, two branches appear, corresponding to positive and negative phase velocities. As earlier, both interpolation and balanced truncation methods feature a damped branch, with a modulated shape near $k \lambda_{D}=0.4$ compared to stock Landau damping. In addition, an unstable branch appears in the $k \lambda_{D}<0.5$ region, which corresponds to the expected bump-on-tail instability. Once again, the balanced truncation method fails in the region $k \lambda_{D}<0.1$. Meanwhile, both interpolation and balanced method outperform both the Hammett-Perkins and the zero-cumulant fluid models for the bump-on-tail instability.

\section{DISCUSSION OF THE METHOD}

\section{A. Back to real space}

The obtained models have been derived in terms of the phase velocity $\zeta=\omega / k$. In order to formulate them in real space, they need to be extended to $k<0$ (the equations for $k=0$ are trivial). The correct way to achieve that is to use the symmetries of the dynamical system. Changing $k \rightarrow-k$ corresponds to discussing frequencies $\omega$ with negative real part. To keep the causality properties, the imaginary part of $\omega$ must remain the same. The transformation rule is then $\omega \rightarrow-\omega^{*}$. The state-space model is

$$
\begin{aligned}
-i \omega x & =A k x+B k \phi & & \text { for } k>0 \\
+i \omega^{*} x & =-A k x-B k \phi & & \text { for } k<0
\end{aligned}
$$

or in general

$$
-i \omega x=|k| \mathfrak{R}[A] x+i k \mathfrak{J}[A] x+|k| \mathfrak{R}[B] \phi+i k \mathfrak{J}[B] \phi(53)
$$

We recover the Hammett-Perkins prescription of a $|k|$ term. In real space, this is associated to a non-local operator instead of a regular $x$-derivative.

\section{B. Application to other kinetic problems}

Since the interpolation method only requires evaluations of the frequency response function, it is very versatile and can be applied to other kinetic problems. For instance, we can consider the linear gyrokinetic problem. Analytical work allows to perform the resonant integrals formally in terms of the Fried and Conte function, leaving smooth integrals in other directions. The KineZero model performs such computations as part of the QuaLiKiz code (0-42 $^{4}$.

An interpolatory reduced model can be formulated to interpolate simultaneously the few first moments of the perturbed distribution function. For instance, the density perturbation $n$ writes

$$
n / \phi=\int \frac{\omega-\omega_{*}}{\omega-k_{\|} v_{\|}-n u_{D}} \mathcal{F} \mathrm{d} v_{\|} \mathrm{d} \mu \mathrm{d} \theta
$$

where $\omega$ is the mode frequency, $n$ its toroidal wave number, $u_{D}$ the toroidal precession angular velocity. $\omega_{*}$ denotes the diamagnetic frequency split into the contributions $\omega_{* n, u, T}$ of the density, parallel velocity and temperature gradients

$$
\omega_{*}=\omega_{* n}+\frac{v_{\|}}{v_{\mathrm{th}}} \omega_{* u}+\omega_{* T}\left(\frac{\frac{m}{2} v_{\|}^{2}+\mu B}{T}-\frac{3}{2}\right)
$$

A reduced model for the response $n / \phi$ can be formulated using three rational functions $H_{* n, u, e}$

$$
\begin{aligned}
n / \phi & =\left(\omega-\omega_{* n}\right) H_{* n}-\omega_{* u} H_{* u}-\omega_{* T}\left(H_{* e}-\frac{3}{2}\right)(56) \\
H_{* n}\left(\frac{\omega}{n}\right) & \approx \int \frac{1}{\omega-k_{\| v_{\|}-n u_{D}}} \mathcal{F} \mathrm{d} v_{\|} \mathrm{d} \mu \mathrm{d} \theta \\
H_{* u}\left(\frac{\omega}{n}\right) & \approx \int \frac{v_{\|} / v_{\text {th }}}{\omega-k_{\|\|_{\|}}-n u_{D}} \mathcal{F} \mathrm{d} v_{\|} \mathrm{d} \mu \mathrm{d} \theta \\
H_{* e}\left(\frac{\omega}{n}\right) & \approx \int \frac{\left(\frac{m}{2} v_{\|}^{2}+\mu B\right) / T}{\omega-k_{\|} v_{\|}-n u_{D}} \mathcal{F} \mathrm{d} v_{\|} \mathrm{d} \mu \mathrm{d} \theta
\end{aligned}
$$

The rational functions $H_{* n, u, e}$ can be built so as to have the same denominator. As such, equation (56) readily translates into a simulable system, and contains a fluid closure.

In this local linear setting, the functions $H_{* n, u, e}$ are independent of the density and temperature gradients. Rather, the gradients only appear in the assembled observables like (56). 
The closure coefficients obtained by this method are independent of the gradients, as seen by Sarazin et al. ${ }^{11}$.

Real-time interpretation of experimental data, especially for future devices such as ITER is a strong motivation to create fast surrogate models based on quasilinear assumptions. Significant speedups have been obtained for instance by ${ }^{43}$ using neural networks trained on a QuaLiKiz database. The same rational functions (57-59) can be used to accelerate the resolution of the gyrokinetic dispersion relation. QuaLiKiz computes the density response $n / \phi$ semi-analytically for several values of the complex eigenfrequency $\omega$, for use in a black-box root-finding algorithm. This procedure can be replaced by the resolution of a polynomial equation in complex space.

$$
1+n^{2} q^{2} \rho_{*}^{2}=\left(\omega-\omega_{* n}\right) H_{* n}-\omega_{* u} H_{* u}-\omega_{* T}\left(H_{* e}-\frac{3}{2}\right)
$$

Extensions of the method for parametric model order reduction $^{44}$ allow to include the dependency on the ballooning angle or the $E \times B$ shear as additional parameters: $H_{* n, u, e}\left(\omega, n, \theta_{b}, \gamma_{E}\right)$. Compared to neural networks predicting the fluxes, this method should require a smaller amount of interpolation data points, while preserving desirable properties like a sharp threshold.

\section{Non-linear extension}

The balanced truncation and interpolation methods are built for linear systems, and their applicability to non-linear systems is not straightforward. However, several methods can be employed to extend the validity of the found closure to the non-linear setting.

The simplest heuristic is to make use of Galilean invariance, replacing the time derivative by the material derivative $\partial_{t} \rightarrow$ $\partial_{t}+\vec{u} \cdot \vec{\nabla}$ (see Mattor and Parker ${ }^{14}$ for instance). A related venue happens by writing the linear closures in terms of linearised cumulants. Under this form, the linearised cumulants can be replaced naturally by their non-linear expression, providing with a non-linear model.

More sophisticated methods have been devised to extend both balanced truncation and interpolation to systems with a so-called bilinear non-linearity ${ }^{34,45,46}$. (Bi-linear refers here to a non-linearity multiplying the state $f$ by the input $\phi$, in opposition to so-called quadratic non-linearity, multiplying $f$ by $f$.) Those methods aim at embedding the non-linearity as products of a fluid moment and the electric field, instead of introducing a quadratic Reynolds stress $\vec{u} \otimes \vec{u}$.

Progress has also been made for fluid systems using balanced proper orthogonal decomposition on nonlinear simulations instead of their linearisation ${ }^{28}$. In the cited works, balancing has shown to improve significantly the relevance of the decompositions. See Goumiri et al. ${ }^{47}$ for an application to plasma physics. Other methods based on neural networks have also shown promising results ${ }^{48}$.

Since the idea is to formulate a reduced order model to accelerate simulations, the interpolation method can be leveraged to compute a reduced model directly in discrete time. This can be done by interpolating at values of $\mathrm{e}^{i \omega \Delta t}$ instead of the frequency ${ }^{38} \omega$. The complexity would not change much everything would still be in a largely opaque matrix-, while the numerical accuracy may benefit.

\section{CONCLUSION}

The reduction of the collisionless Vlasov equation has been investigated using the balanced truncation method. This method constructs a simplified dynamical space, whose basis is chosen so as to maximise both the reachability and the observability of its individual vectors. The rapid decay of the Hankel singular values indicate a strong reducibility of the Vlasov model. The balanced truncation reduced models reconstruct the Vlasov dynamics accurately in the thermal phase velocity region, but have degraded performances in the asymptotic low and high phase velocity regimes. In particular, the energy wrongly flows from the particles to the wave in the high phase velocity regime. This violation comes from the difficulty to approximate the Landau damping rate $\omega^{4} / k^{3} \exp \left(-\omega^{2} / k^{2}\right)$ as a rational function. The rational approximation found by balanced truncation overshoots to negative values. As a result, the coupled Vlasov-Poisson dynamics features a spurious instability at high phase velocities, which makes it unusable as a fluid closure. General positivity-preserving model reduction is still an open problem ${ }^{22}$, but existing algorithms may allow more systematic application of the method.

We have formulated a novel non-collisional closure method based on interpolation of the linear response function, and applied it to the Vlasov-Poisson problem. The method generalises derivations based on asymptotic matching at low and high frequency. The resulting model outperforms usual Hammett and Perkins' formulation in the thermal phase velocity range, for both the density response, the Landau damping rate and the bump-on-tail instability growth rate. The general method is very versatile, and should allow for efficient and cheap model order reduction for gyrokinetic toroidal drift waves in the collisionless regime.

\section{ACKNOWLEDGEMENTS}

The authors want to thank Ch. Passeron, D. Zarsozo, and acknowledge stimulating discussions with the participants at the 2019 Festival de Théorie in Aix-en-Provence. This work has been carried out within the framework of the EUROfusion Consortium and was supported by the EUROfusion - Theory and Advanced Simulation Coordination (E-TASC) initiative under the TSVV (Theory, Simulation, Verification and Validation) "L-H transition and pedestal physics" project (20192020). It has also received funding from the Euratom research and training program. The authors gratefully acknowledge funding from the European Commission Horizon 2020 research and innovation program under Grant Agreement No. 824158 (EoCoE-II). The views and opinions expressed herein do not necessarily reflect those of the European Commission. This work was also granted access to the HPC resources of TGCC and CINES made by GENCI, and to the EUROfu- 
sion High Performance Computer Marconi-Fusion. We acknowledge PRACE for awarding us access to Joliot-Curie at GENCI@CEA, France and MareNostrum at Barcelona Supercomputing Center (BSC), Spain. The data that support the findings of this study are available from the corresponding author upon reasonable request.

\section{REFERENCES}

${ }^{1}$ A. M. Dimits, G. Bateman, M. A. Beer, B. I. Cohen, W. Dorland, G. W. Hammett, C. Kim, J. E. Kinsey, M. Kotschenreuther, A. H. Kritz, L. L. Lao, J. Mandrekas, W. M. Nevins, S. E. Parker, A. J. Redd, D. E. Shumaker, R. Sydora, and J. Weiland, "Comparisons and physics basis of tokamak transport models and turbulence simulations," Physics of Plasmas 7, 969983 (2000).

${ }^{2}$ G. W. Hammett and F. W. Perkins, "Fluid moment models for landau damping with application to the ion-temperature-gradient instability," Physical Review Letters 64, 3019-3022 (1990).

${ }^{3}$ Z. Chang and J. D. Callen, "Unified fluid/kinetic description of plasma microinstabilities. Part I: Basic equations in a sheared slab geometry," Physics of Fluids B: Plasma Physics 4, 1167-1181 (1992).

${ }^{4}$ P. B. Snyder, G. W. Hammett, and W. Dorland, "Landau fluid models of collisionless magnetohydrodynamics," Physics of Plasmas 4, 3974-3985 (1997).

${ }^{5}$ T. Passot and P. L. Sulem, "Long-alfvén-wave trains in collisionless plasmas. ii. a landau-fluid approach," Physics of Plasmas 10, 3906-3913 (2003).

${ }^{6}$ P. Goswami, T. Passot, and P. L. Sulem, "A landau fluid model for warm collisionless plasmas," Physics of Plasmas 12, 102109 (2005), http://arxiv.org/abs/physics/0509091v1.

${ }^{7}$ T. Chust and G. Belmont, "Closure of fluid equations in collisionless magnetoplasmas," Physics of Plasmas 13, 012506 (2006).

${ }^{8}$ H. Sugama, T.-H. Watanabe, and W. Horton, "Collisionless kinetic-fluid closure and its application to the three-mode ion temperature gradient driven system," Physics of Plasmas 8, 2617-2628 (2001).

${ }^{9}$ H. Sugama, T.-H. Watanabe, and W. Horton, "Comparison between kinetic and fluid simulations of slab ion temperature gradient driven turbulence," Physics of Plasmas 10, 726-736 (2003).

${ }^{10}$ Smith, "Dissipative closures for statistical moments, fluid moments, and subgrid scales in plasma turbulence," (1997).

${ }^{11}$ Y. Sarazin, G. Dif-Pradalier, D. Zarzoso, X. Garbet, P. Ghendrih, and V. Grandgirard, "Entropy production and collisionless fluid closure," Plasma Physics and Controlled Fusion 51, 115003 (2009).

${ }^{12}$ P. Hunana, G. P. Zank, M. Laurenza, A. Tenerani, G. M. Webb, M. L. Goldstein, M. Velli, and L. Adhikari, "New closures for more precise modeling of landau damping in the fluid framework," Physical Review Letters 121 (2018), 10.1103/physrevlett.121.135101, http://arxiv.org/abs/1809.05718v1.

${ }^{13}$ P. Hunana, A. Tenerani, G. P. Zank, M. L. Goldstein, G. M. Webb, E. Khomenko, M. Collados, P. S. Cally, L. Adhikari, and M. Velli, "An introductory guide to fluid models with anisotropic temperatures. part 2. kinetic theory, padé approximants and landau fluid closures," Journal of Plasma Physics 85 (2019), 10.1017/s0022377819000850.

${ }^{14}$ N. Mattor and S. E. Parker, "Nonlinear kinetic fluid equations," Physical Review Letters 79, 3419-3422 (1997).

${ }^{15}$ L. Wang, B. Zhu, X.-Q. Xu, and B. Li, "A Landau-fluid closure for arbitrary frequency response," AIP Advances 9, 015217 (2019).

${ }^{16} \mathrm{C}$. Mullis and R. Roberts, "Synthesis of minimum roundoff noise fixed point digital filters," IEEE Transactions on Circuits and Systems 23, 551562 (1976).

${ }^{17}$ B. Moore, "Principal component analysis in linear systems: Controllability, observability, and model reduction," IEEE Transactions on Automatic Control 26, 17-32 (1981).

${ }^{18}$ S. Gugercin and A. C. Antoulas, "A survey of model reduction by balanced truncation and some new results," International Journal of Control 77, 748766 (2004).

${ }^{19}$ S. Gugercin, A. Antoulas, and C. Beattie, "A rational Krylov iteration for optimal H2 model reduction," in Proceedings of MTNS, Vol. 2006 (2006).
${ }^{20} \mathrm{C}$. Beattie and S. Gugercin, "Realization-independent $\mathcal{H}_{2}$-approximation," in 2012 IEEE 51 st IEEE Conference on Decision and Control (CDC) (IEEE, 2012).

${ }^{21}$ S. Gugercin, "Model reduction via interpolation," (2017).

${ }^{22}$ S. Grivet-Talocia and B. Gustavsen, Passive macromodeling (John Wiley \& Sons, Inc, Hoboken, New Jersey, 2016).

${ }^{23}$ B. Fried and S. Conte, "The plasma dispersion function academic," (1961).

${ }^{24}$ L. C. Goodrich, "General recurrence relation for use in evaluating moments of the integrand of the plasma dispersion function," Physics of Fluids 15, 715 (1972).

${ }^{25}$ N. G. Lehtinen, "Error functions," Tech. Rep. (2010).

${ }^{26}$ I. Jolliffe, Principal Component Analysis (Springer-Verlag GmbH, 2002).

${ }^{27}$ K. Pearson, "LIII. On lines and planes of closest fit to systems of points in space," Philosophical Magazine Series 6 2, 559-572 (1901).

${ }^{28} \mathrm{C}$. W. Rowley, "Model reduction for fluids, using balanced proper orthogonal decomposition," International Journal of Bifurcation and Chaos 15, $997-$ 1013 (2005).

${ }^{29}$ T. Penzl, "Algorithms for model reduction of large dynamical systems," Linear Algebra and its Applications 415, 322-343 (2006).

${ }^{30} \mathrm{~B}$. Gustavsen and A. Semlyen, "Rational approximation of frequency domain responses by vector fitting," IEEE Transactions on Power Delivery 14, 1052-1061 (1999).

${ }^{31}$ A. C. Antoulas, C. A. Beattie, and S. Gugercin, "Interpolatory model reduction of large-scale dynamical systems," in Efficient Modeling and Control of Large-Scale Systems (Springer US, 2010) pp. 3-58.

${ }^{32}$ C. Beattie and S. Gugercin, "Model reduction by rational interpolation," in Model Reduction and Approximation (Society for Industrial and Applied Mathematics, 2017) pp. 297-334, arxiv:1409.2140.

${ }^{33}$ A. Mayo and A. Antoulas, "A framework for the solution of the generalized realization problem," Linear Algebra and its Applications 425, 634-662 (2007).

${ }^{34} \mathrm{I}$. V. Gosea, Model order reduction of linear and nonlinear systems in the Loewner framework, Ph.D. thesis (2017).

${ }^{35}$ I. V. Gosea and A. C. Antoulas, "Approximation of a damped EulerBernoulli beam model in the Loewner framework," (2017), 1712.06031v1.

${ }^{36}$ I. Pontes Duff Pereira, Large-scale and infinite dimensional dynamical model approximation, Ph.D. thesis (2017).

${ }^{37}$ D. S. Karachalios, I. V. Gosea, and A. C. Antoulas, "Data-driven approximation methods applied to non-rational functions," PAMM 18 (2018), 10.1002/pamm.201800368.

${ }^{38} \mathrm{P}$. Vuillemin and C. Poussot-Vassal, "Discretisation of continuous-time linear dynamical model with the Loewner interpolation framework," (2019), 1907.10956v1.

${ }^{39}$ T. Reis and T. Stykel, "Positive real and bounded real balancing for model reduction of descriptor systems," International Journal of Control 83, 74-88 (2009).

${ }^{40}$ C. Bourdelle, X. Garbet, G. T. Hoang, J. Ongena, and R. Budny, "Stability analysis of improved confinement discharges: internal transport barriers in tore supra and radiative improved mode in TEXTOR," Nuclear Fusion 42, 892-902 (2002).

${ }^{41}$ J. Citrin, C. Bourdelle, F. J. Casson, C. Angioni, N. Bonanomi, Y. Camenen, X. Garbet, L. Garzotti, T. Görler, O. Gürcan, F. Koechl, F. Imbeaux, O. Linder, K. van de Plassche, P. Strand, and G. S. and, "Tractable fluxdriven temperature, density, and rotation profile evolution with the quasilinear gyrokinetic transport model QuaLiKiz," Plasma Physics and Controlled Fusion 59, 124005 (2017).

${ }^{42}$ C. Stephens, "Qualikiz: An analytic derivation," Tech. Rep. (DIFFER, 2019).

${ }^{43}$ K. L. van de Plassche, J. Citrin, C. Bourdelle, Y. Camenen, F. J. Casson, V. I. Dagnelie, F. Felici, A. Ho, and S. V. M. and, "Fast modeling of turbulent transport in fusion plasmas using neural networks," Physics of Plasmas 27, 022310 (2020).

${ }^{44}$ S. Lefteriu, A. C. Antoulas, and A. C. Ionita, "Parametric model reduction in the Loewner framework," IFAC Proceedings Volumes 44, 12751-12756 (2011).

${ }^{45}$ P. Benner and T. Damm, "Lyapunov equations, energy functionals, and model order reduction of bilinear and stochastic systems," SIAM Journal on Control and Optimization 49, 686-711 (2011).

${ }^{46} \mathrm{P}$. Benner and T. Breiten, "Interpolation-based $\boldsymbol{H}_{2}$-model reduction of bilinear control systems," SIAM Journal on Matrix Analysis and Applications 
33, 859-885 (2012).

${ }^{47}$ I. R. Goumiri, C. W. Rowley, Z. Ma, D. A. Gates, J. A. Krommes, and J. B. Parker, "Reduced-order model based feedback control of the modified hasegawa-wakatani model," Physics of Plasmas 20, 042501 (2013).

${ }^{48}$ C. Ma, B. Zhu, X.-Q. Xu, and W. Wang, "Machine learning surrogate models for Landau fluid closure," Physics of Plasmas 27, 042502 (2020). 